\title{
Postmenopausale Frauen: Lungenkrebsrisiko unter Hormontherapie?
}

\section{Verschiedenste Risiken der kombi- nierten Hormonbehandlung sind bekannt. Im letzten Jahr wurde die Liste weiter ergänzt: Offenbar erhöht die Substitution auch das Risiko, an Lungenkrebs zu sterben.}

- Die beiden doppelblinden placebokontrollierten Hormontherapie-Studien im Rahmen der Womens Health Initiative (WHI) waren 2002 bzw. 2004 aufgrund einer insgesamt negativen Nutzen-Risiko-Relation vorzeitig gestoppt worden. Eine neue Auswertung der Daten hatte 2009 ergeben, dass unter Östrogen plus Gestagen zwar nicht die Inzidenz, jedoch die Sterberate am Lungenkarzinom erhöht wurde, wenn diese auch, in absoluten Zahlen betrachtet, im Verum- wie Placeboarm sehr gering war.

Nun untersuchte man die Daten von Patientinnen unter einer Östrogenmonotherapie. Über 10000 hysterektomierte postmenopausale Frauen (50-79 Jahre) hatten 0,625 mg konjugiertes Östrogen täglich oder Placebo erhalten. Ih- re Daten wurden daraufhin untersucht, ob Östrogen allein die Inzidenz oder die Mortalität eines Lungenkarzinoms beeinflusst.

Nach median 7,9 Jahren Follow-up war unter der Hormontherapie bei 61 Frauen ein Lungenkarzinom diagnostiziert worden, in der Placebogruppe bei 54 (Inzidenzrate 0,15 resp. $0,13 \%$ pro Jahr; Hazard Ratio: 1,17; keine Signifikanz). Nicht kleinzellige Karzinome kamen in beiden Studienarmen in ähnlicher Zahl vor; auch Stadium und Grading waren vergleichbar. Ebenso wichen die Todesraten durch Lungenkarzinome (34 bzw. 33 Fälle) praktisch nicht voneinander ab. Gleiches traf auf die Sterblichkeit durch andere Ursachen nach der Lungenkrebsdiagnose zu.

Anders als die kombinierte Hormontherapie (konjugiertes Östrogen plus Gestagen), die die Mortalität durch Lungenkrebs erhöhte, wirkte sich in dieser aktuellen Post-hoc-Analyse das Östrogen allein nicht ungünstig auf die Inzidenz und die Mortalität eines Lungenkarzinoms aus.

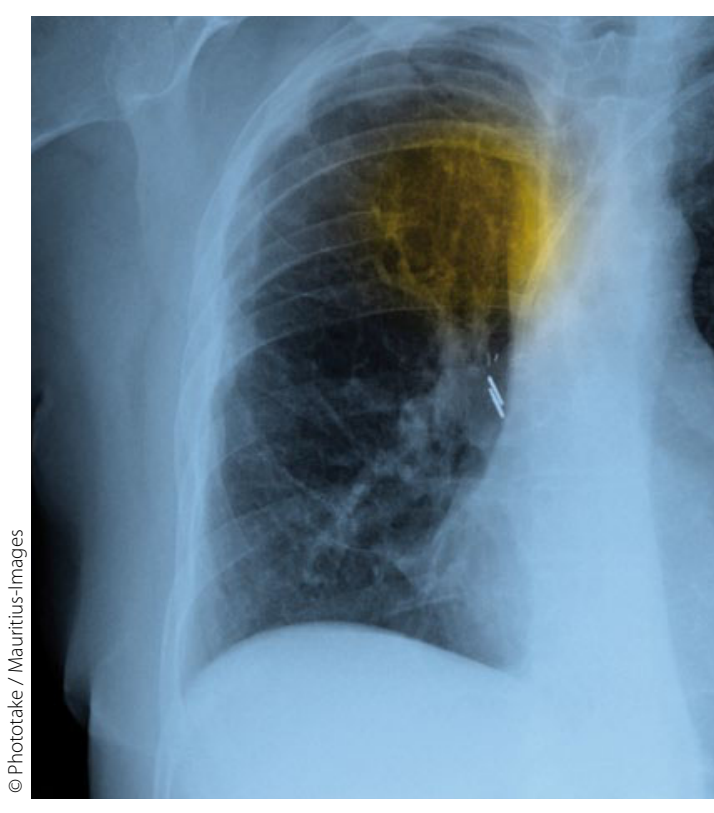

$60 \%$ der Nichtraucher mit Lungenkrebs sind Frauen.

- R. T. Chlebowski et al.

Lung cancer among postmenopausal women treated with estrogen alone in the Women's Health Initiative randomized trial. J. Natl. Cancer Inst. 102 (2010) 1413-1421

\section{Kommentar}

Möglicherweise zeigen uns die Ergebnisse der WHI-Studie, dass nicht die Östrogen-, sondern die Gestagenkomponente der Hormonersatztherapie (HET) den kanzerogenen Effekt bezüglich der Entwicklung von Lungenkarzinomen haben könnte.

Insgesamt scheinen Hormone einen eher geringen Einfluss auf die Lungenkrebspathogenese zu haben. Es existieren unterschiedliche beziehungsweise gegensätzliche Informationen bezüglich der Rolle der weiblichen Hormone beim Lungenkarzinom.

Folgende widersprüchliche Beobachtungen zu Geschlechtsunterschieden beim Lungenkarzinom wurden gemacht:

- Nicht rauchende Frauen scheinen häufiger zu erkranken als nicht rauchende Männer. 20\% der Frauen mit Lungenkrebs sind Nichtraucher, $60 \%$ der Nichtraucher mit Lungenkrebs sind Frauen.

_ Im Lungengewebe von rauchenden Frauen finden sich höhere Spiegel an nikotinassoziierten DNA-Addukten als bei Männern, eventuell begründet dies eine höhere Entartungswahrscheinlichkeit bei Frauen. _ Überlebensanalysen von Lungenkrebsstudien, besonders in frühen Stadien, ergaben häufig ein besseres Überleben der Frauen. - Bei Frauen (und Nichtrauchern) kommen die prognostisch günstigeren aktivierenden EGFR (Rezeptor für den epithelialen
Wachstumsfaktor)-Genmutationen häufiger als bei Männern vor. - Korrelationen zwischen der Östrogenrezeptor (ER)-Expression auf Tumorzellen und der EGFR-Mutation bei Adenokarzinomen der Lunge werden vermutet.

- An Lungenkrebszelllinien wurden die Östrogenrezeptoren alpha (ERa) und beta (ERß) nachgewiesen.

- In Untersuchungen in China sah man bei nie rauchenden Frauen einen protektiven Effekt durch einen höheren Hormoneinfluss (späte Menopause, längere protektive Periode, höhere Geburtenzahl) gegenüber der Entwicklung eines Lungenkarzinoms.

_ Diskutiert werden Interaktionen zwischen Sexualhormonen, Steroidrezeptoren, metabolischen Enzymen und der Signaltransduktion im Bereich der Regulation von Zellwachstum und der Apoptose.

Fazit: Der Einfluss der weiblichen Hormone und speziell der HET auf das Lungenkrebsrisiko bleibt spekulativ. Die HET sollte nur bei eindeutiger Indikation und so niedrig und so kurz dosiert wie möglich eingesetzt werden, wobei die wesentlichen Risiken nicht der Lungenkrebs, sondern vaskuläre Risiken bleiben. Der wichtigste Risikofaktor für den Lungenkrebs ist das Zigarettenrauchen und der effektivste Schutz davor bleibt das Nicht-Rauchen.

M. SERKE 